\title{
Enhanced resolution of lossy interferometry by coherent amplification of single photons
}

\author{
Chiara Vitelli, ${ }^{1,2}$ Nicolò Spagnolo, ${ }^{1,2}$ Lorenzo Toffoli, ${ }^{1}$ Fabio Sciarrino, $, 1,3$, , and Francesco De Martini ${ }^{1,4}$ \\ ${ }^{1}$ Dipartimento di Fisica, Sapienza Università di Roma, piazzale Aldo Moro 5, I-00185 Roma, Italy \\ ${ }^{2}$ Consorzio Nazionale Interuniversitario per le Scienze Fisiche della Materia, piazzale Aldo Moro 5, I-00185 Roma, Italy \\ ${ }^{3}$ Istituto Nazionale di Ottica, largo Fermi 6, I-50125 Firenze, Italy \\ ${ }^{4}$ Accademia Nazionale dei Lincei, via della Lungara 10, I-00165 Roma, Italy
}

\begin{abstract}
In the quantum sensing context most of the efforts to design novel quantum techniques of sensing have been constrained to idealized, noise-free scenarios, in which effects of environmental disturbances could be neglected. In this work, we propose to exploit optical parametric amplification to boost interferometry sensitivity in the presence of losses in a minimally invasive scenario. By performing the amplification process on the microscopic probe after the interaction with the sample, we can beat the losses detrimental effect on the phase measurement which affects the single-photon state after its interaction with the sample, and thus improve the achievable sensitivity.
\end{abstract}

PACS numbers: 42.50.Ex, 42.50.Dv, 42.50.St

The aim of quantum sensing is to develop methods to extract the maximum amount of information from a system with a minimal disturbance on it. Indeed, the possibility of performing precision measurements by adopting quantum resources can increase the achievable precision going beyond the semiclassical regime of operation [13]. In the case of interferometry, this can be achieved by the use of the so-called N00N states, which are quantum mechanical superpositions of just two terms, corresponding to all the available photons $N$ placed in either the signal arm or the reference arm. The use of N00N states can enhance the precision in phase estimation to $1 / N$, thus improving the scaling of the achievable precision with respect to the employed resources [4, 5]. This approach can have wide applications for minimally invasive sensing methods in order to extract the maximum amount of information from a system with minimal disturbance. The experimental realization of protocols involving N00N states containing up to 4 photons have been realized in the last few years [6 10]. Other approaches [11, 12 have focused on exploiting coherent and squeezed light to generated fields which approximate the features of N00N states. Nevertheless, these quantum states turn out to be extremely fragile under losses and decoherence 13], unavoidable in experimental implementations. A sample, whose phase shift is to be measured, may at the same time introduce high attenuation. Since quantum-enhanced modes of operations exploit fragile quantum mechanical features the impact of environmental effects can be much more deleterious than in semiclassical schemes, destroying completely quantum benefits [14, 15]. This scenario puts the beating of realistic, noisy environments as the main challenge in developing quantum sensing. Very recently, the theoretical and experimental investigations of quantum states of light have attracted much attention, leading to the best possible precision in optical two-mode interferometry, even in the presence of experimental imperfections 16 21].

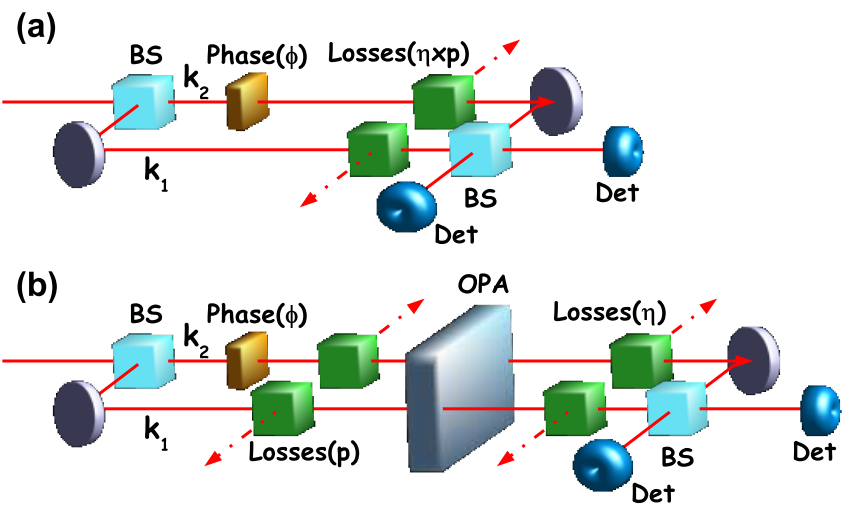

FIG. 1: Scheme for the phase measurement. (a) Interferometric scheme adopted to estimate the phase $(\phi)$ introduced in the mode $k_{2}$. (b) Interferometric scheme adopting a single photon and the optical parametric amplifier: the amplification of the single photon state is performed before dominant losses.

In this work, we adopt a hybrid approach based on a high gain optical parametric amplifier operating for any polarization state in order to transfer quantum properties of different microscopic quantum states in the macroscopic regime 22, 23]. By performing the amplification process of the microscopic probe after the interaction with the sample we can beat the losses detrimental effect on the phase measurement which affects the singlephoton state after the sample. Our approach can be adopted in a minimally invasive scenario where a fragile sample, such as biological or artifacts systems, requires as few photons as possible impinging on it in order to prevent damages. The action of the amplifier, i.e., the process of optimal phase covariant quantum cloning, is to broadcast the phase information codified in a single photon into a large number of particles. Such multiphoton states have been shown to exhibit a high resilience 
to losses 24 26] and can be manipulated by exploiting a detection scheme which combines features of discrete and continuous variables. The effect of losses on the macroscopic field consists in the reduction of the detected signal and not in the complete cancellation of the phase information as would happen in the single-photon probe case, thus improving the achievable sensitivity. This improvement does not consist in a scaling factor but turns out to be a constant factor in the sensitivity depending on the optical amplifier gain. Hence, the sensitivity still scales as the $\sqrt{N}$, where $N$ is the number of photons impinging on the sample, but the effect of the amplification process is to reduce the detrimental effect of losses by a factor proportional to the number of generated photons.

Let us review the adoption of single photons in order to evaluate the unknown phase $\varphi$, Fig.1-(a). The phase $\varphi$ introduced in the path $k_{2}$ is probed by sending to the sample $N$ input photons, each one in the state $2^{-1 / 2}\left(|1\rangle_{k 1}+|1\rangle_{k 2}\right)$. After the propagation, the sample introduces a phase $\varphi$ on the probe beam and each photon is found in the state: $\frac{1}{\sqrt{2}}\left(|1\rangle_{k 1}+e^{i \varphi}|1\rangle_{k 2}\right)$. The two modes $k_{1}$ and $k_{2}$ are then combined on a beamsplitter $(B S)$ and detected by $\left(D_{1}^{\prime}, D_{2}^{\prime}\right)$ with an overall detection efficiency equal to $t$. $N$ performed experiments leads to an output signal equal to $I=I\left(D_{1}^{\prime}\right)-I\left(D_{2}^{\prime}\right)=t N \cos \varphi$, whose fluctuations are given by $\sigma=(t N)^{1 / 2}$. The uncertainty on the phase measurement around the value $\frac{\pi}{2}$ can hence be estimated as $\Delta \varphi=\left(\frac{\partial I}{\partial \varphi}\right)^{-1} \triangle I=\frac{1}{\sqrt{t N}}$, the semiclassical shot noise limit, and the sensitivity of the interferometer can be evaluated as $S_{1 \text { phot. }}=\frac{1}{\Delta \varphi}=\sqrt{t N}$.

In order to avoid the detrimental effect of a low value of $t$, our strategy involves the amplification of the singlephoton probe, Fig.1-(b). In the theory and experiment described here, the two modes $k_{1}$ and $k_{2}$ correspond to two orthogonal polarization modes: horizontal $(H)$ and vertical $(V)$ associated to the same longitudinal spatial mode $k$. The input single photon is prepared in the polarization state: $|+\rangle=\frac{1}{\sqrt{2}}(|H\rangle+|V\rangle)$. After the propagation over the interferometer, the photon acquires the unknown phase $\varphi:|\varphi\rangle=\frac{1}{\sqrt{2}}\left(|H\rangle+e^{\imath \varphi}|V\rangle\right)$. The amplification performed by the optical parametric device generates the output state $\left|\Phi^{\varphi}\right\rangle=\widehat{U}_{O P A}|\varphi\rangle=$ $\cos \frac{\varphi}{2}\left|\Phi^{+}\right\rangle+\imath \sin \frac{\varphi}{2}\left|\Phi^{-}\right\rangle$, where $\left|\Phi^{+,-}\right\rangle$are the wavefunctions described in Ref. [24]. Precisely, the state $\left|\Phi^{+}\right\rangle$ $\left(\left|\Phi^{-}\right\rangle\right)$presents a Planckian probability distribution as a function of photons polarized $\vec{\pi}_{-}\left(\vec{\pi}_{+}\right)$and a long tail distribution as a function of photons polarized $\vec{\pi}_{+}\left(\vec{\pi}_{-}\right)$. The two distributions belonging to the state $\left|\Phi^{+}\right\rangle$and $\left|\Phi^{-}\right\rangle$partially overlap, but become distinct on the border of the Fock states plane [27]. For the state $\left|\Phi^{\varphi}\right\rangle$, the average number of photons emitted over the polarization mode $\vec{\pi}_{+}$is equal to $\left\langle n_{+}\right\rangle=\bar{n}+\cos ^{2} \frac{\varphi}{2}(2 \bar{n}+1)$ with $\bar{n}=\sinh ^{2} g$ and $g$ the gain of the amplifier, while the average number of photons emitted over the polarization mode $\vec{\pi}_{-}$is equal to $\left\langle n_{-}\right\rangle=\bar{n}+\sin ^{2} \frac{\varphi}{2}(2 \bar{n}+1)$. The previous expressions lead to a phase-dependent intensity with a visibility $V=\frac{\left\langle n_{+}\right\rangle-\left\langle n_{-}\right\rangle}{\left\langle n_{+}\right\rangle+\left\langle n_{-}\right\rangle} \rightarrow 0.50$ for $g \rightarrow \infty$. The resilience to losses of such multiphoton fields [25] renders them suitable for the implementation of quantum information applications in which noisy channels and low detection efficiency are involved. We consider the case in which the losses are unavoidable during the detection process, and happen after the single-photon amplification (Fig 1). After the propagation over a lossy channel, the state evolves from $\left|\Phi^{\varphi}\right\rangle\left\langle\Phi^{\varphi}\right|$ into a mixed state $\hat{\rho}_{\eta}^{\varphi}$. For details on the explicit expressions of the coefficients of the density matrix $\hat{\rho}_{\eta}^{\varphi}$, see [25].

After the amplification stage and the transmission losses, the received field is analyzed through singlephoton detectors $\left(D_{1}^{\prime}, D_{2}^{\prime}\right)$ in the $\left\{\vec{\pi}_{+}, \vec{\pi}_{-}\right\}$polarization basis. Our aim is to compare the achievable sensitivity with and without the optical amplifier $(g=0)$. To take into account experimental imperfections, we divide the losses $t$ in two contributions: the first one includes all the losses between the sample and the optical amplifier $(p)$, while the second parameter takes into account all the inefficiencies up to the detection stage $(\eta): t=p \times \eta$. Our strategy cannot compensate for losses that occur before the amplifier $(p)$, but can compensate for large (even very large, if the gain is high enough) losses after the amplification $(\eta)$. A first insight on this property of the optical parametric amplifier has been given in Ref. [28] by analyzing the signal-to-noise of the amplification of a coherent state signal in lossy conditions. The sensitivity $S_{a m p l}$, obtained by measuring the difference $\langle D\rangle=\left\langle n_{+}\right\rangle-\left\langle n_{-}\right\rangle$ intensity signals provided by the detectors around the phase value $\varphi=\frac{\pi}{2}$, is found to be:

$$
S_{a m p l .}=\frac{\sqrt{N} p \eta c}{\left\{\eta^{2}[p \bar{n}(4 c+2)+2 \bar{n} c]+\eta[p c+2 \bar{n}]\right\}^{1 / 2}}
$$

with $c=2 \bar{n}+1$.

Let us first consider the case $p=0.5$ : Fig.2-(a) reports the logarithm of the enhancement of the squared sensitivity $E=\left(\frac{S_{\text {ampl. }}}{S_{1 \text { phot. }}}\right)^{2}$ versus $g$ and $\eta . E$ represents the reduction factor in the number of photons sent onto the sample in order to obtain the same information on the phase $\varphi$, by exploiting the amplification strategy with respect to the single-photon probe scheme. As it can be observed in Fig.2-(a), a large improvement can be obtained in the regime of high losses and large gain of the amplifier. The motivation of such behavior is the following: the present approach allows us to increase the number of detected photons by a factor $4 \bar{n}$ with respect to the single-photon case keeping a visibility of the fringe patterns reduced only to $50 \%$ (for $g \rightarrow \infty$ ). The enhancement $\mathrm{E}$ is then slightly affected by the reduction in the visibility, due to the amplification noise [29], while it is significantly improved by the increase in the detected signal. 
(a)

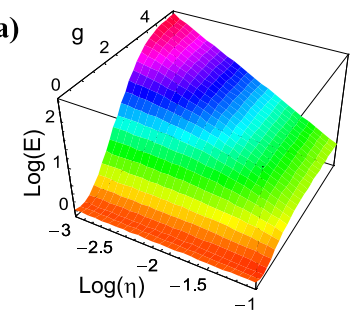

(b)

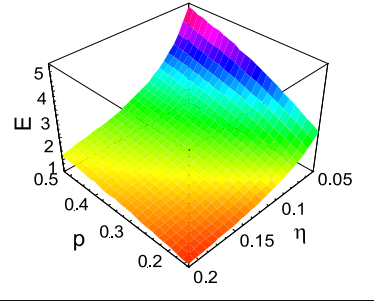

(d)

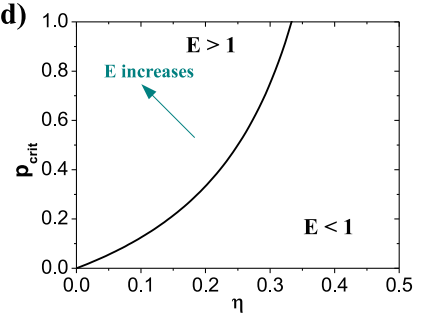

FIG. 2: (a) Logarithm of the enhancement versus the nonlinear gain $g$ and the transmittivity of the lossy channel $\eta$ for a value of losses between the phase-shifter and the amplifier equal to $p=0.5$. (b) Trend of $E$ as a function of losses before amplification $0.15 \leq p \leq 0.5$ and after amplification $0.05 \leq \eta \leq 0.2(g=4.5)$. (c) Contour plot of the enhancement as a function of the logarithm of $p$ and $\eta$. The lighter region corresponds to $E>1$, the darker one to $E<1$. (d) Non ideal case $p \neq 1$ : trend of the injection probability critical value for which $E>1$ as a function of the detection efficiency.

In Fig.2-(b) we report the trend of the enhancement as a function of losses $0.15 \leq p \leq 0.5$ and $0.05 \leq \eta \leq 0.2$ for a nonlinear gain of $g=4.5$. Such a range corresponds to typical values of losses and detection efficiency achievable in practical schemes. We observe that also in this regime an enhancement greater that 1 can be obtained. For large values of the gain $g$ the enhancement saturates to the value: $E_{l i m}=\frac{p}{\eta(2 p+1)}$ [contour plot in Fig.2-(c)]; we can then identify a critical value of $p$ above which the enhancement is greater than 1: $p_{\text {crit }}=\frac{\eta}{1-2 \eta}$. For $\eta \geq 0.33$ no enhancement can be achieved by exploiting the amplification strategy (see figure 2-(d)).

The previous theoretical predictions have been experimentally tested by adopting a high gain optical parametric amplifier with a maximum gain $g=4.5$. A detailed description of the apparatus can be found in Ref. [24, 27]. The single-photon probe was generated in a first nonlinear crystal and heralded by a trigger detector $D_{T}$; hence, the phase shifting $\varphi$ was introduced via a Soleil-Babinet compensator. Then the probe photon was superposed to an ultraviolet pump beam and injected into a second nonlinear crystal realizing the optical parametric amplifier. The output radiation was then coupled with a single mode fiber and detected with single-photon detectors. Additional controlled losses were introduced by adopting neutral optical filters. For the sake of simplicity we propose working in the single-photon counting regime; in order to describe the detection apparatus in a linear regime, the following condition for the average number of detected photons must be satisfied $\eta\left\langle n_{ \pm}\right\rangle<<1$ (experimental details can be found in the supplementary material [30]). We found experimentally a value of $p$ equal to 0.15 due to spatial and spectral mismatch between the injected single photon and the ultraviolet pump beam $\left(k_{p}^{\prime}\right)$. The output fringe patterns have been recorded for different values of the gain $g$ and hence of the generated number of photons in the amplifier. In the extreme condition with $\eta=3 \times 10^{-4}$ and $g=4.5$ we observed an enhancement of a factor $\sim 210$, as shown in Fig 3 (a), in which is reported the trend of $E$ as a function of the amplifier gain, compared with the theoretical prediction.

We now discuss the optimality of the measurements performed on the multiphoton state, in order to extract the maximum information about the phase $\varphi$ codified in the optical field. This quantity is expressed by the quantum Fisher information [3, 31], defined as $H(\varphi)=$ $\operatorname{Tr}\left[\hat{\rho}^{\varphi} \hat{L}_{\varphi}\right]$, where $\hat{L}_{\varphi}$ is the symmetric logarithmic derivative $\partial_{\varphi} \hat{\rho}^{\varphi}=\frac{\hat{L}_{\varphi} \hat{\rho}^{\varphi}+\hat{\rho}^{\varphi} \hat{L}_{\varphi}}{2}$ and $\hat{\rho}^{\varphi}$ is the density matrix of the state in which the phase is codified. The quantum Cramer-Rao bound [3] quantifies the maximum precision achievable on the estimation of the phase $\varphi$ optimized over all possible measurements as: $\Delta^{2} \varphi \geq 1 / H(\varphi)$. In the high lossy regime $\eta\left\langle n_{ \pm}\right\rangle \ll 1$, the single-photon amplified states lead to a quantum Fisher information equal to $H_{a m p l}(\varphi) \approx 2 \bar{n} \eta p\left(1+p^{-1}\right)^{-1}$, to be compared with the single-photon case, which gives $H_{1 p h o t}(\varphi)=\eta p$. This result allows us to investigate the optimality of the counting measurement strategy. The sensitivity achieved with this scheme, given by Eq.(1), can be written in the high lossy regime as $S_{a m p l}^{2}=\left(\Delta^{2} \varphi\right)^{-1} \approx 2 \bar{n} \eta p\left(1+p^{-1}\right)^{-1}$, thus saturating the Cramer-Rao bound and ensuring the optimality of this scheme in the high lossy regime.

As a more sophisticated strategy, it is possible to elaborate on an approach which leads to higher visibility of
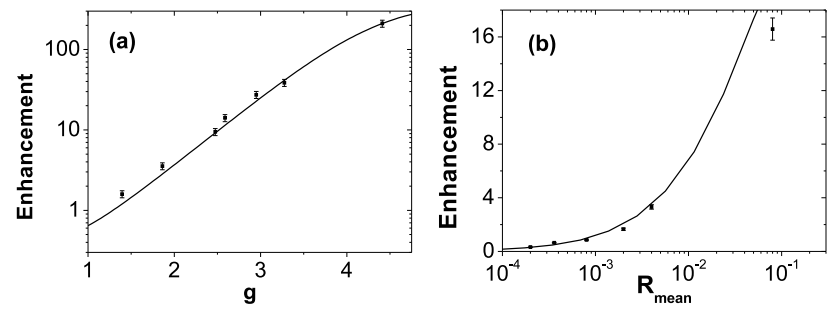

FIG. 3: (a) Experimental results of the enhancement $E$ versus the nonlinear gain for the counting detection strategy. Continuous line: theoretical prediction for the expected enhancement with $\eta=3 \times 10^{-4}, p=0.15$. (b) Experimental results of the enhancement $E$ versus the signal rate, the continuous line reports the theoretical prediction for $p=0.14$, $\eta=0.005$. 
the detected fringe patterns at the cost of a reduced detection rate of the signal: the output radiation is measured in polarization with two linear detectors, for instance photomultipliers. The intensity signals generated by the detectors proportional to the orthogonally polarized number of photons are compared shot by shot by the orthogonality-filter (OF) electronic device introduced in Ref. [24]. When the number of photons $m_{\varphi}$, detected in the $\vec{\pi}_{\varphi}$ polarization, exceeds $n_{\varphi_{\perp}}$, detected in the $\vec{\pi}_{\varphi \perp}$ polarization, over a certain adjustable threshold $k$, i.e. $m_{\varphi}-n_{\varphi_{\perp}}>k$, the $(+1)$ outcome is assigned to the event and the state $\left|\Phi^{\varphi}\right\rangle$ is detected. On the contrary, when the condition $n_{\varphi_{\perp}}-m_{\varphi}>k$ is satisfied, the (-1) outcome is assigned and the state $\left|\Phi^{\varphi \perp}\right\rangle$ is detected. Finally, an inconclusive result $(0)$ is obtained when the unbalance between detected pulses does not exceed the threshold $k$. As the gain is increased, the number of transmitted photons $\eta\langle n\rangle$ becomes sufficient to detect all the $N$ repeated trials. In the high losses regime, at variance with the single-photon case, all pulses can be exploited to extract information about the phase $\varphi$. The action of the OF is then to select those events that can be discriminated with higher fidelity, leading to an increase in the visibility, at the cost of discarding part of the data. According to these considerations, the "detection" efficiency of the scheme, i.e. the percentage of detected events, is given by the average signal $\bar{\eta}=R_{\text {mean }}(k)$ filtered by the OF device. This parameter $\bar{\eta}$ corresponds to the overall efficiency of the amplification-OFbased detection scheme. We calculated the phase measurement uncertainty through the standard definition $\Delta \varphi_{O F}=\Delta R_{O F}(\varphi)\left|\frac{\partial R_{O F}(\varphi)}{\partial \varphi}\right|^{-1}$. The minimum uncertainty is achieved for $\varphi=\frac{\pi}{2}$. The resulting sensitivity averaged over $N$ trials is thus $S_{O F}=V \sqrt{R_{\text {mean }}} \sqrt{N}$, where $V$ is the visibility of the fringe pattern. This expression shows that the phase fluctuations no longer depend on the efficiency $\eta$ of the channel, but only on the average percentage of detected pulses $R_{\text {mean }}$.

We have experimentally tested the enhancement obtained by the OF strategy. We report in Fig. 3-(b) the experimental trend of the enhancement as a function of the signal rate compared with the expected theoretical trend ( $p=0.14, \eta=0.005)$. In the adopted apparatus the single-photon fringe pattern shows a visibility $\sim 50 \%$ due to the generation of more than a single-photon pair by the first nonlinear crystal adopted as the heralded single-photon source. This seed visibility value is also responsible for a reduction of the amplified state visibility and has been taken into account in the comparison between the two strategies. By comparing the enhancement obtained through the counting and the OF-based detection methods we can conclude that the first one allows us to achieve a higher enhancement.

In conclusion, the ability to generate suitable quan- tum light probes and quantum detectors is a crucial prerequisite for the operation of any quantum sensor. The optimal probes maximizing the sensitivity and performance of the sensors can be theoretically determined, but the resulting quantum states are often very complicated, difficult to generate, and extremely sensitive to losses and noise. We proposed and realized a simple conceptual strategy to apply in a lossy scenario that can be engineered with the existing quantum-optics technology. Our results show that a large sensitivity improvement can be achieved even in a high losses condition in which the dominant losses act after the interaction of the probe with the sample, hence including all the inefficiencies in the detection of the probe (spatial and spectral filtering, transmission, efficiency of the detectors).

We acknowledge support by the "Futuro in Ricerca" Project HYTEQ, and Progetto d'Ateneo of "Sapienza" Università di Roma.

* Electronic address: fabio.sciarrino@uniroma1.it

[1] V. Giovannetti et al., Science 306, 1330 (2004).

[2] V. Giovannetti et al., Phys. Rev. Lett. 96, 010401 (2006).

[3] C. W. Helstrom, Quantum Detection and Estimation Theory (Academic Press, New York, 1976).

[4] A. N. Boto et al., Phys. Rev. Lett. 85, 2733 (2000).

[5] J. P. Dowling, Contemp. Phys. 49, 125 (2008).

[6] M. D'Angelo et al., Phys. Rev. Lett. 87, 013602 (2001).

[7] P. Walther et al., Nature 429, 158 (2004).

[8] M. W. Mitchell et al., Nature 429, 161 (2004).

[9] H. S. Eisenberg et al., Phys. Rev. Lett. 94, 090502 (2005).

[10] T. Nagata et al., Science 316, 726 (2007).

[11] T. Ono et al., Phys. Rev. A 81, 033819 (2010).

[12] I. Afek et al., Science 328, 879 (2010).

[13] G. Gilbert et al., J. Mod. Opt. 55, 3283 (2008).

[14] M. A. Rubin et al., Phys. Rev. A 75, 053805 (2007).

[15] A. Shaji et al., Phys. Rev. A 76, 032111 (2007).

[16] S. Huver et al., Phys. Rev. A 78, 063828 (2008).

[17] U. Dorner et al., Phys. Rev. Lett. 102, 040403 (2009).

[18] L. Maccone et al., Phys. Rev. A 79, 023812 (2009).

[19] R. Demkowicz-Dobrzanski et al., Phys. Rev. A 80, 013825 (2009).

[20] M. Kacprowicz et al., Nature Photonics, 4, 357 (2010).

[21] T.-W. Lee et al., Phys. Rev. A 80, 063803 (2009).

[22] F. De Martini, Phys. Rev. Lett. 81, 2842 (1998).

[23] F. De Martini, Phys. Lett. A 250, 15 (1998).

[24] F. De Martini et al., Phys. Rev. Lett. 100, 253601 (2008).

[25] F. De Martini et al., Phys. Rev. A 79, 052305 (2009).

[26] F. De Martini et al., Phys. Rev. Lett. 103, 100501 (2009).

[27] E. Nagali et al., Phys. Rev. A 76, 042126 (2007).

[28] J. A. Levenson et al., Quantum Semiclass. Opt. 9, 221 (1997).

[29] B. R. Mollow et al., Phys. Rev. 160, 1076 (1966); Phys. Rev. 160, 1097 (1966).

[30] Supplementary Information.

[31] M. G. A. Paris, Int. J. Quant. Inf. 7, 125 (2009). 


\title{
Supplementary informations: Enhanced resolution of lossy interferometry by coherent amplification of single photons
}

\author{
Chiara Vitelli, ${ }^{1,2}$ Nicolò Spagnolo, ${ }^{1,2}$ Lorenzo Toffoli, ${ }^{1}$ Fabio Sciarrino, $, 1,3$, : and Francesco De Martini ${ }^{1,4}$ \\ ${ }^{1}$ Dipartimento di Fisica, "Sapienza" Università di Roma, piazzale Aldo Moro 5, I-00185 Roma, Italy \\ ${ }^{2}$ Consorzio Nazionale Interuniversitario per le Scienze Fisiche della Materia, piazzale Aldo Moro 5, I-00185 Roma, Italy \\ ${ }^{3}$ Istituto Nazionale di Ottica Applicata, largo Fermi 6, I-50125 Firenze, Italy \\ ${ }^{4}$ Accademia Nazionale dei Lincei, via della Lungara 10, I-00165 Roma, Italy
}

\section{EXPERIMENTAL IMPLEMENTATION OF THE PROTOCOL}

We address in more details the experimental implementation of the proposed metrology scheme: figure 1. The single photon probe is conditionally generated by a BBO non linear crystal (C1) on spatial mode $\mathbf{k}_{1}$ together with an entangled photon on spatial mode $\mathbf{k}_{T}$.
The overall state produced by $C 1$ is expressed by: $\left|\psi^{-}\right\rangle=$ $\frac{1}{\sqrt{2}}\left(|H\rangle_{T}|V\rangle_{1}-|V\rangle_{T}|H\rangle_{1}\right)$, and is obtained through the process of spontaneous parametric down-conversion, consisting in the annihilation of a pump photon at wavelength (wl) $\lambda_{p}=397.5 \mathrm{~nm}$ followed by the creation of twin photons, orthogonally polarized, at wl $\lambda=795 \mathrm{~nm}$.
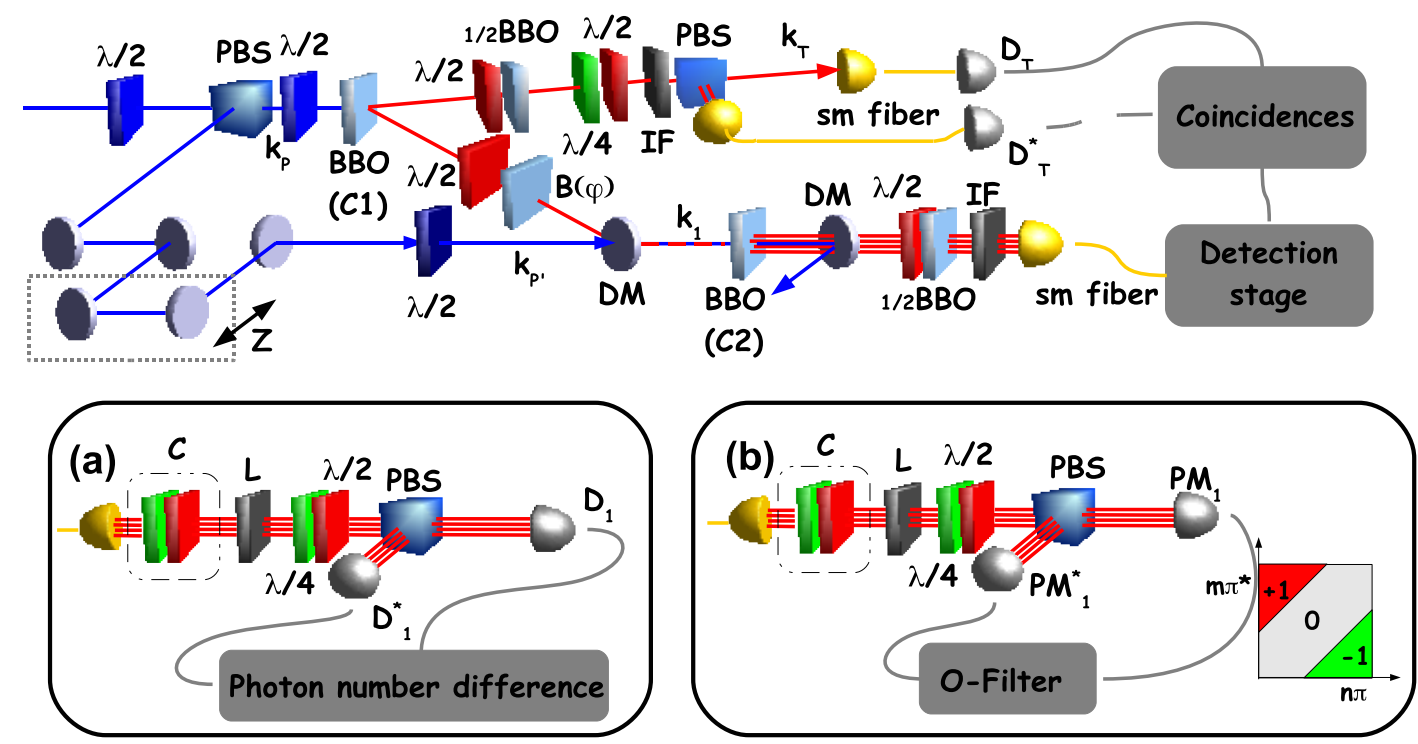

FIG. 1: Experimental setup of the QI-OPA based interferometric scheme. An intense pulsed pump beam of $P=750 \mathrm{~mW}$ and $\lambda=397.5 \mathrm{~nm}$ is generated by a Ti:Sa laser system. The pump beam is split between modes $\mathbf{k}_{p}$ and $\mathbf{k}_{p}^{\prime}$. In the first BBO crystal (C1), a single photon $|+\rangle_{1}$ state is prepared upon detection on mode $\mathbf{k}_{T}$ with a single-photon SPCM-AQR14 detector $\left(D_{T}\right)$. Then, the phase $\varphi$ is introduced through a Babinet-Soleil compensator (B) and the probe state $|\varphi\rangle$ after the interaction is then injected in the QI-OPA and superimposed with the pump beam on mode $\mathbf{k}_{p}^{\prime}$. Spatial and temporal matching are obtained through an adjustable delay line (Z). After the amplification process has occured, spectral and spatial filtering are performed through the insertion of an interference filter (IF) and the coupling with a single-mode fiber. The signal is then sent to the detection apparatus. (a) Measurement scheme based on detection of the average of the photon number difference $\hat{D}=\hat{n}_{+}-\hat{n}_{-}$. After fiber polarization compensation (C), the output field undergoes controlled losses up to the single-photon level throughout a tunable attenuator $(\mathrm{L})$. Then, the field is analyzed in polarization and detected with two single-photon SPCM-AQR14 detectors $\left(D_{1}, D_{1}^{*}\right)$ (b) O-Filter based detection scheme. After fiber polarization compensation (C), the field is analyzed in polarization and detected with two photomultipiers $\left(P M_{1}, P M_{1}^{*}\right)$. The photocurrents are shot-by-shot processed by an electronic device (O-Filter), whose action is described in the text and in Ref.[1].

The overall pump beam is generated by a Ti:Sa laser system. At a first stage, a pulsed 250 fs beam with a repetion rate of $75 \mathrm{MHz}$ at wavelength $\lambda=795 \mathrm{~nm}$ is gen- 
erated Ti:Sa modelocked MIRA900, pumped by a Verdi V5 Nd:Yag solid state laser. The output beam from the MIRA900 is injected into the Ti:Sa REGA9000 amplifier, pumped by a Verdi V18 with an optical power set to $14 \mathrm{~W}$. The overall laser system allows to obtain a 1.5 $\mathrm{W}$ output beam at wavelength $\lambda=795 \mathrm{~nm}$, that, after a double harmonic generation process, generates the experiment pump beam at wl $\lambda_{p}=397.5 \mathrm{~nm}$ of power $P=750 \mathrm{~W}$. This beam is split into two parts with different optical powers; the weaker one impinges into $C 1$ and generates the entangled state $\left|\psi^{-}\right\rangle$, the stronger one is directed towards the amplifier crystal $C 2$ and is superimposed spatially and temporally with the single photon probe after the interaction with the sample (simulated by a Soleil-Babinet compansator $B(\varphi)$, which allows to insert a tunable phase shift between the $\vec{\pi}_{H}$ and the $\vec{\pi}_{V}$ polarization, in figure 11). The number of photons generated in the amplification process depends exponentially on the nonlinear gain of the amplifier $g$. The maximum value of $g$ experimentally found is $g_{\max }=4.5$. The value of the nonlinear gain has been measured by exploiting the method of Ref.[2, 3], i.e. by measuring the dependence of the counts rate on the incident pump power in the spontaneous regime and by fitting the curve with the expected functional form $C=\frac{\eta \tanh ^{2} g}{1-(1-\eta) \tanh ^{2} g}$ [Fig 2 .

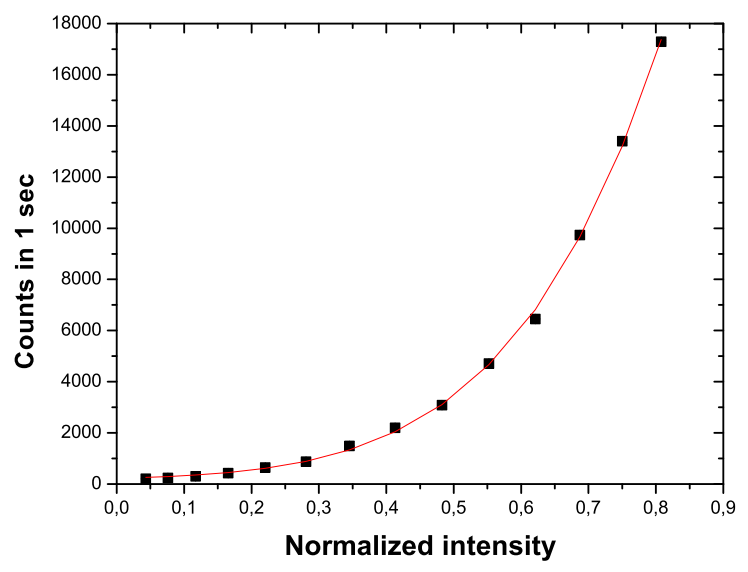

FIG. 2: Trend of counts detected by $D_{1}, D_{1}^{*}$ as a function of the normalized intensity signal impinging on the amplifier, working in a spontaneous (not-injected) configuration.

\section{High losses regime and single-photon detection}

In this section we investigate the first measurement strategy implemented in our experiment [Fig@(a)]. The amplified state, after spectral and spatial filtering, is deliberately attenuated up to the single-photon level. The signal is then analyzed in polarization and detected by two single photon counting modules SPCM, $D_{1}$ and $D_{1}^{*}$ in figure 1- (a). The resulting signals, triggered by the click of detectors $\left\{D_{T}, D_{T}^{*}\right\}$ on mode $\mathbf{k}_{T}$, are hence subtracted and the difference in the number of photons $\hat{D}$ is recorded as a function of the phase $\varphi$, inserted by the babinet $B(\varphi)$ on the probe path.
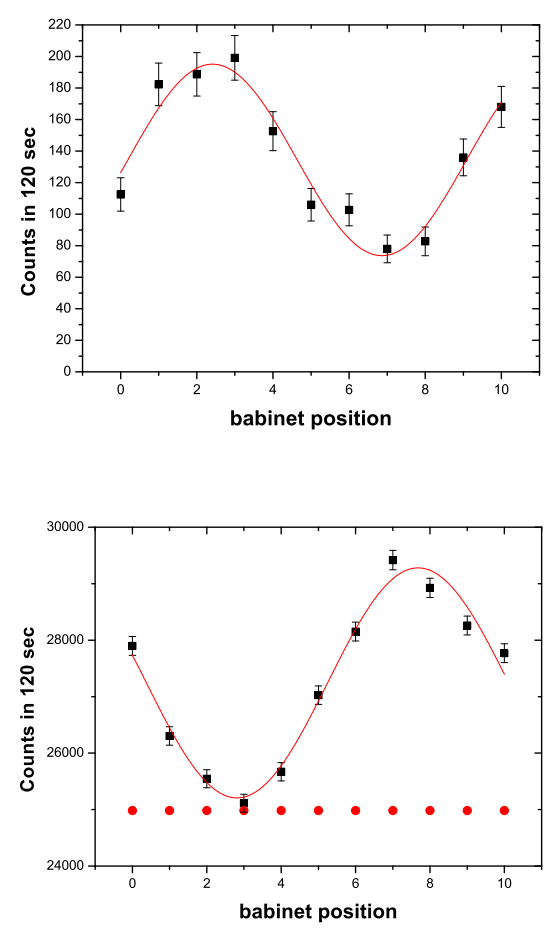

FIG. 3: Experimental fringe pattern for the single photon probe (left) and for the amplified beam (right).

The fringe patterns obtained in absence and in presence of the amplifier are reported in Fig $[3$, in order to compare the performances of the two phase-estimation schemes. In the first case the obtained visibility is $V_{1 p h o t}=0.45$, whose value differs from the expected unitary one due to the generation of more than a single photon pair by the first nonlinear crystal, adopted as the heralded single photon source. This value of the seed visibility is also responsible for a reduction of the amplified state visibility $V_{a m p l}\left(g_{\max }\right)=0.08$ and has been taken into account in the comparison between the two strategies.

\section{Increased resilience to losses through an orthogonality filter}

In this section we investigate an alternative measurement strategy apt to obtain an enhancement in the resilience to losses of the interferometric estimation of an unknown phase. The presented method is based on an electronic discriminator, the orthogonality filter(OF) de- 
vice introduced in Ref. [1], which performs a dichotomic threshold detection, allowing to increase the visibility of the amplified signal with respect to the SPCM-based strategy at the cost of a lower detection rate.

The action of the $\mathrm{OF}$ is then to select those events which can be discriminated with higher fidelity, leading to an increase in the visibility. The latter operation is achieved at the cost of discarding a part of the data. According to these considerations, the quantum efficiency of the scheme is only given by the average signal $\bar{\eta}=R_{\text {mean }}(k)$ filtered by the OF device. This parameter $\bar{\eta}$ takes the role of the overall efficiency of the OF-based scheme. The sensitivity, found through the standard definition, is: $\frac{1}{\Delta \varphi}=\frac{1}{\Delta I_{O F}(\varphi)}\left|\frac{\partial I_{O F}(\varphi)}{\partial \varphi}\right|$. That can be further symplified obtaining the expression:

$$
S_{O F}=\frac{1}{\Delta \varphi_{O F}}=\frac{\left|\sin \varphi\left(I_{\max }-I_{\min }\right)\right|}{\left(\left(I_{\max }-I_{\min }\right) \cos ^{2} \frac{\varphi}{2}+I_{\min }\right)^{1 / 2}}
$$

The minimum is obtained in $\varphi=\frac{\pi}{2}$, where the sensitivity can be put in the form:

$$
S_{O F}=\frac{1}{\Delta \varphi_{O F}}=V \sqrt{R_{\text {mean }}}
$$

Finally, the average over $N$ trials gives an improvement of $\sqrt{N}$ :

$$
S_{O F}=V \sqrt{R_{\text {mean }}} \sqrt{N}
$$

This expression shows that the sensitivity $S_{O F}$ does not depend on the efficiency $\eta$ of the channel, but only on the average filtered signal $R_{\text {mean }}$.

The OF based strategy has been experimentally implemented by adopting at the detection stage two photomultipliers, $P M_{1}$ and $P M_{1}^{*}$ in figure 1-(b). The two intensity signals, proportional to the orthogonally polarized number of photons, are compared shot-by-shot by the orthogonality-filter that renders at its output two TTL signals. Coincidences of such signals with the corresponding click of the detector on trigger mode $\left(D_{T}\right.$ or $D_{T}^{*}$ ) are recorded. By increasing the discrimination threshold of the OF we can achieve a higher visibility for the amplified field respect to the one obtained through the SPCM based strategy of previous section. In figure 4 is reported the fringe pattern obtained with a percentage of detected signal equal to $3.6 \times 10^{-4}$, the resulting visibility is $\sim 0.53$. The trend of the visibility as a function of the percentage of detected pulses is reported in figure 5, compared with the theoretical prediction for $p=0.14$ and $\eta=0.005$. We observe that a higher visibility is obtained for a lower count rate. By comparing the fringe pattern in figure 4 with the one in figure 3-(b), we observe that the amplified field visibility is increased by a factor $\sim 7$ but the counts rate is decreased by a factor 30 . It is worth noting that the two detection strategies implemented in this paper (single-photon

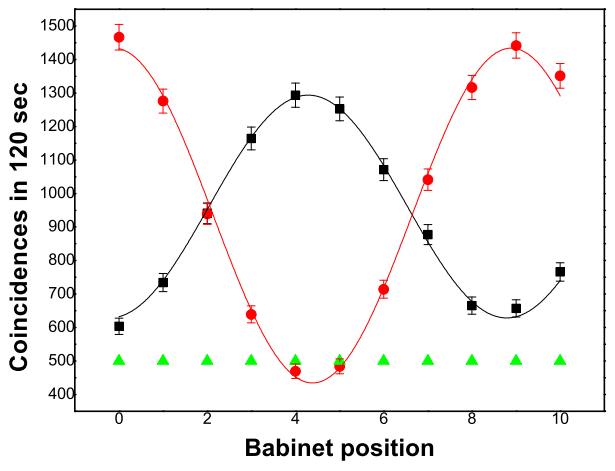

FIG. 4: Experimental fringe pattern for a percentage of detected signal equal to $3.6 \times 10^{4}$, the obtained visibility is $\sim 0.53$.

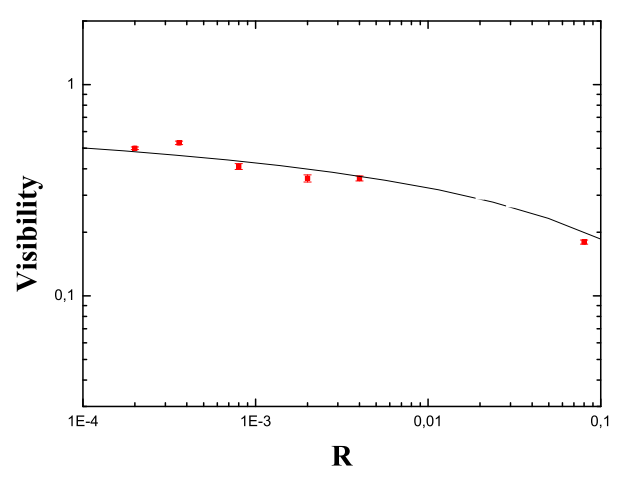

FIG. 5: Experimental trend of visibility as a function of the percentage of filtered signal, the continuous line reports the theoretical prediction for $p=0.14, \eta=0.005$.

vs OF) have been investigated in two different regimes. For the SPCM-based strategy, a tunable attenuator has been exploited to achieve the condition $\eta \bar{n} \sim 0.1$, corresponding in our experiment to a value of the overall detection efficiency of $\eta \sim 3 \times 10^{4}$. For the OF detection method, the overall efficiency $\eta$ is due to spectral filtering, single-mode fiber coupling, transmission losses and detection efficiency of the photomultipliers, leading to an overall value of $\eta \sim 0.005$.

* Electronic address: fabio.sciarrino@uniroma1.it

[1] F. De Martini, F. Sciarrino, and C. Vitelli, Phys. Rev. Lett. 100, 253601 (2008).

[2] H. S. Eisenberg, G. Khoury, G. A. Durkin, C. Simon, and D. Bouwmeester, Phys. Rev. Lett. 93, 193901 (2004)

[3] M. Caminati, F. De Martini, R. Perris, F. Sciarrino, and V. Secondi, Phys. Rev. A 73, 032312 (2006) 